\title{
Struktur und Funktion der Festung Torgau im 19. Jahrhundert
}

\author{
Uwe Niedersen
}

Die Festung Torgau war im 19. Jahrhundert wie folgt belegt: ab 1810 diente sie als sächsische Landesfestung, etwas später dann, 1813, kam sie unter französisches Kommando, 1814 wurde sie von der preußischen Armee besetzt, und 1815 fiel sie mit der Abtretung des sächsischen Gebiets an das Königreich Preußen. 1889 erfolgte die Aufhebung der Festung.

Wie eine Festung von ihrer Gestalt her ausgelegt wird und wofür sie danach in Gebrauch steht, entscheiden militärische, technische, ökonomische, vor allem finanzielle sowie politische und auch zivile Faktoren, ja überhaupt die Interessenlage des jeweiligen Staates. Die Planungs- und Baugeschichte der Festung Torgau zeigt das eindrücklich.

\section{Sächsische und französische Festungsbaupläne}

Kursachsen wurde 1806 Mitglied in dem durch Napoleon Bonaparte (1769-1821) dominierten Rheinbund und quasi mit gleichem Federstrich zum Königreich erhoben. Andere Rheinbundmitglieder wie Bayern, Württemberg gaben zu erkennen, dass Sachsen dabei seine Selbstständigkeit nicht aufgeben sollte, sondern diese erhalten und als Mittelmacht nicht in Neutralität verharren möge. Vielmehr habe Sachsen als ein souveränes Mitglied des Rheinbundes aufzutreten.

Das Ingenieur-Korps, hier ein Zeichnung um 1800, trug dunkelgrüne Röcke mit roten Kragen und Aufschlägen; rote Hosen sowie schwarze Hüte mit silbernen Agraffen. Die Galauniform (2) trug man zu festlichen Anlässen; die Dienstuniform (3) war das Alltagskleid des Offiziers; der Interimsrock (1) wurde außer Dienst getragen wurde. ๑ Förderverein Europa Begegnungen e.V.

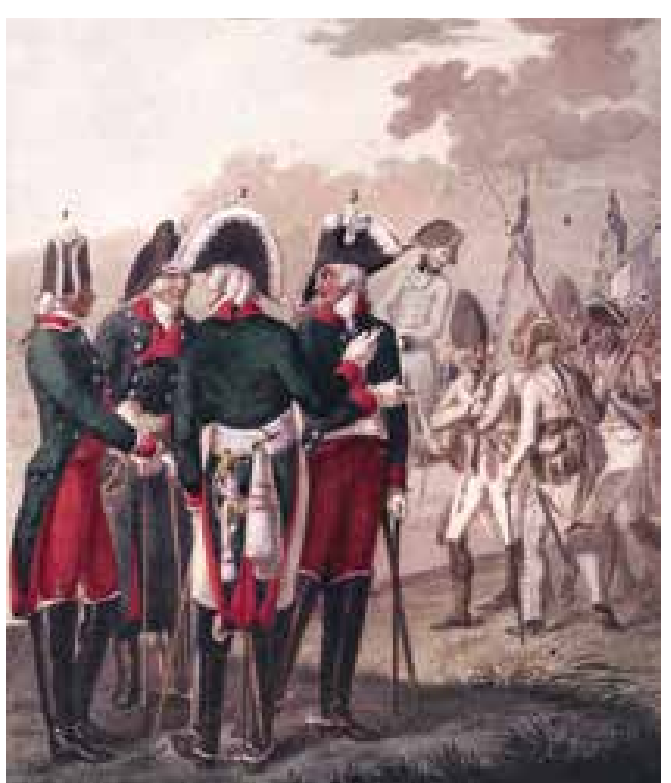

Ingenieur-Offiziere der sächsischen Armee wie Ernst Ludwig Aster (1778-1855), Johann August von Le Coq (1748-1828) und Johann Georg Lehmann (1765-1811) forderten selbstbewusst Militärreformen. Sie meinten, in der neuen Rheinbund-Konstellation müsse es, was Sachsen betrifft, jetzt darum gehen, eine Politik der „aktiven“, eben nicht der ungeschützten Neutralität zu betreiben: Johann Georg Lehmann schrieb: „Eine gute Armee, unterstützt durch gute Festungen, mit allen nötigen Kriegsmitteln reichlich versehen, sind die kräftigsten Mittel zu diesem Zwecke zu gelangen, wenn sie ein weises Ministerium handhabten. Die Zeitumstände waren niemals günstiger als jetzt [1810/11], um Sachsens Selbstständigkeit zu begründen.“

Was das Königreich Sachsen damals im Besonderen benötigte, war eine zentral gelegene Landesfestung, die als Rückzugs- und Zufluchtsort der ganzen sächsischen Armee, als Depot für Ausrüstung und Proviant sowie als Verwahrungsort für Akten, Schriften und Gelder des Hofes dienen sollte. Der Generalstab entschied sich um 1809/10, das im Königreich Sachsen recht zentral und geostrategisch günstig gelegene Torgau zur Landesfestung auszubauen. Damit sollten frühere Fehler und Versäumnisse der kursächsischen Politik unwiederholbar ausgemerzt werden. Sachsen drängte förmlich danach, aus der bitteren Niederlage Lehren zu ziehen, welche einst der Preußenkönig Friedrich II. (1712-1786) 1756 dem Land beigebracht hatte, als er die gesamten sächsischen Truppen, die auf einer Plateaufläche bei Pirna ungedeckt standen und auf die Österreicher als Retter warteten, in Gefangenschaft geführt hatte.

Napoleon schrieb Sachsen nach der Schlacht bei Jena und Auerstedt eine militärische Rolle bei seiner offensiv geführten europäischen Eroberungspolitik zu. Das gerade erst entstandene Königreich Sachsen hielt sich zwar betont zurück, irgendwelche durch Napoleon eroberten östlichen Gebiete, etwa in Polen, regieren zu wollen, doch der französische Kaiser übertrug den Sachsen eine Art Mitherrschaft über das durch Eroberung neu gebildete Herzogtum Warschau. Das war aber ein Gebiet preußischer, russischer und auch österreichischer Interessen. Sehr schnell konnte die dort stationierte sächsische Armee aus diesem Gebiet wieder verdrängt werden. Carl Friedrich Wilhelm von Gersdorff (1765-1829), der Chef des sächsischen 
Generalstabes, „preiste“ in einem Vortrag mögliche Niederlagen der Sachsen im Herzogtum Warschau mit ein und fragte sich, wo dann die sächsische Armee Halt, Deckung, ja Zuflucht finden könne: „Benötigt wird ein Ort, der Positionen für die Armee darbietet, die sich unter dem Schutz und mit Deckung der Festung zu sammeln hätte. Die Festung Torgau müsse Vereinigungspunkt und Aufnahme einer aus dem Herzogtum Warschau sich zurückziehenden Armee sein. Dorthin müsse unser Augenmerk gehen, gegen die Oder müsse es gewendet sein“. Gersdorff hatte mit dieser Einschätzung die Landesfestung Torgau als Versammlungs- bzw. Zufluchtsort der sächsischen Armee definiert. Aus dieser Funktion heraus hatte sich die Struktur der neuen Festung Torgau abzuleiten. Dem Rat kompetenter Ingenieur-Offiziere folgend, legte der sächsische Generalstab fest, vor der Hauptfestung, gemeint war die zentrale Stadtfestung Torgau, einen Außenring aus Schanzen und Erdwerken anzulegen: mit einer Lünette bei Repitz, mit Fort Mahla und Fort Zinna, einer Windmühlenschanze an der neuen Straße nach Leipzig, eine Teichschanze, einer weiteren Schanze Richtung Loßwig bei der Ratsziegelei und schließlich noch einer Schanze am Färberdamm, nahe Loßwig. Ostwärts der Elbe waren um den Brückenkopf ebenfalls ein Ring von Verschanzungen (Redouten) und zusätzlich zwei Werke (Lünetten) bei Werdau und Zwethau vorgesehen. Zwischen diesen äußeren Werken und der Stadt als Hauptfestung würde sich sogleich ein „verschanztes Lager“ bilden, in welchem Tausende sächsische Soldaten im Verbund mit der Kernfestung einen Versammlungs- bzw. Rückzugsort vorfinden, um offensiv oder defensiv handeln zu können.

Torgau hatte sich über die Jahrhunderte in seiner Entwicklung vor allem Richtung Westen ausgedehnt. Die dabei entstandenen Vorstädte mit Waisenhaus, Hospital und Spitalkirche sollten in einem 10-Bastionen-Festungsumlauf (Enceinte) mit einbezogen werden und so erhalten bleiben. Der Ingenieur-Hauptmann Ernst Ludwig Aster plante außerdem bombenfeste Schutzräume. In dem Memoire, das er in Paris am 2. November $1810 \mathrm{Na}$ poleon vorlegte, war der Bau eines Doppelwalls für die Hauptangriffsfront im Nordwesten, gegliedert in einen Haupt- und einen Niederwall, beide durch einen Graben getrennt und zugleich mit kasemattierten Schutzräumen bzw. kasemattierten Feuerstellungen in den Wallflanken versehen, enthalten. In Sachsen war festgelegt worden, dass die sächsische Elb- und Landesfestung Torgau ein permanenter Befestigungskomplex werden solle. Torgau sollte auch in Friedenszeiten eine dauerhaft unterhaltene Anlage bleiben. Diesem Gedanken widersprach Napoleon. Er benötigte damals alles andere als ein nach Selbständigkeit strebendes Sachsen, noch dazu eine durch das sächsische Militär flankierte, eigenständige Neutralitätspolitik. Prinzipiell hatte er kein Interesse daran, in Sachsen eine dauerhafte, baulich ausgedehnte und anspruchsvolle Landesfestung errichten zu lassen. Was er wollte, war eine offensiv ausgerichtete Festung.

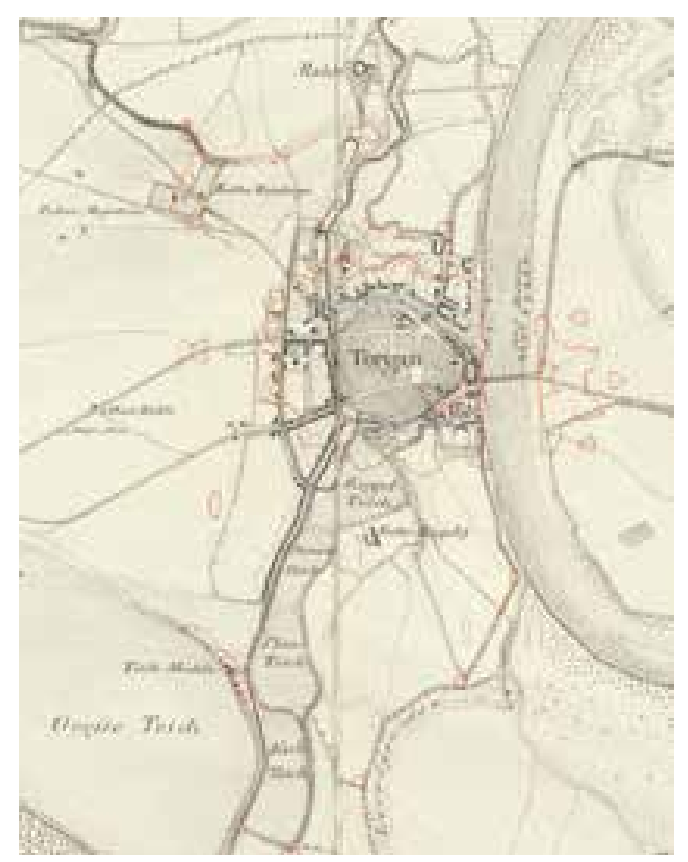

Entwurf einer 10-BastionenLandesfestung Torgau nach Le Coq, Aster, Lehmann, 1810. Dem hier angedachten 10-Bastionen-Umzug ist ein Plan Torgaus von 1770 unterlegt. ๑ Förderverein Europa Begegnungen e.V.
Dort, wo Napoleon war, konzentrierten sich die Armeen, da wurden Flüsse überquert, da wurde aufmarschiert. Mit einer straff organisierten Beweglichkeit der Truppenteile ging es in die Feldschlachten. Es wurde geschossen, gefochten, erobert, anfangs auch noch gesiegt. Das System „Napoleon“ bestand und funktionierte nur in der Bewegung. Es waren die fortlaufenden Offensiven, in den militärischen wie auch in den zivilen Bereichen, die den Bestand des Systems garantierten. Bewegung war Eroberung anderer Länder und das anhaltende Ausplündern der besetzten Gebiete.

Napoleon befahl, die Elb- und Landesfestung Torgau als Kampagne-Festung auszubauen, also als eine Fortifikation, die die Offensive der Feldtruppen unterstützen und als „aktives“ Element wirken sollte. Die primäre Funktion der durch Napoleons Bestimmung neu auszulegenden sächsischen Festung Torgau bestand vor allem darin, einen sicheren Flussübergang an der mittleren Elbe zu garantieren und die Beweglichkeit der Truppen, besonders an Flussübergängen, sicherzustellen. Die Sachsen, die selbstbewusst in den Rheinbund eingetreten waren, erlebten mit einer gänzlichen Neubestimmung der Funktion der sächsischen Festung Torgau eine grundsätzliche Niederlage, und der sächsische König Friedrich August I. (1750-1827) unternahm nicht den Versuch, Napoleon zu widersprechen. Somit war bereits ab 1810 sichtbar geworden, dass sich Sachsen lediglich als Mitläufer Napoleons sah, entfernt davon, eigenes Neutralitätsbestreben $\mathrm{zu}$ behaupten. Friedrich August I. hielt dann auch bis zum bitteren Ende an Napoleon fest.

Napoleon hatte bereits 1810/11 Russland zum nächsten Objekt seiner Eroberungen bestimmt. Da der Kaiser aber durchaus bedenken musste, dass sich die Russland-Kampagne auch zu einer Niederlage entwickeln könnte, was ja dann auch wirklich geschah, kalkulierte er in seine Überlegungen Rückmärsche mit ein. Daher waren funktionieren- 
Plan der sächsischen Elb- und Landesfestung Torgau mit Hauptwerk und Außenwerken (Brückenkopf, Fort Zinna, Fort Mahla, vier Elbe-Lünetten), Situation vom März 1813. Das ist daran erkennbar, weil durch das noch bestehende Waisenhaus die Spitze des Ravelins vor dem Leipziger Tor baulich noch nicht vollendet werden konnte. ๑ Förderverein Europa Begegnungen e.V. de, stabile Elbübergänge von Wichtigkeit. Auch war dem Kaiser der Franzosen bewusst, dass nach einem Rückzug aus Russland Feldschlachten wahrscheinlich auf sächsischem Gebiet stattfinden und prinzipielle Entscheidungen erbringen würden. Napoleon musste auch zudem mit mehreren verbündeten europäischen Armeen als Gegner rechnen. Der Franzose wollte sie alle schlagen, indem er versuchte, den Armeen im jeweils separaten Kampf die Niederlage zu bereiten. Er war der Ansicht, dass das mit einer höchst beweglichen Armee durchführbar sein müsse.

Dafür entwickelte Napoleon eine neue Taktik. Die Elbübergänge sollten nun nicht mehr einfach von West nach Ost oder irgendwann auch wieder einmal umgekehrt, von Ost nach West, erfolgen. Nein, jetzt hatten die Truppen in einem schnelleren Takt und in einem über längere Sicht nicht vorhersehbaren Hin und Her die Flüsse zu überqueren.

Napoleons neue Taktik basierte auf dem Konzept des „doppelten Brückenkopfes“. Ein Brückenkopf ist eine Befestigung auf dem feindwärtigen Ufer vor einem sicher und beständig $\mathrm{zu}$ haltenden Flussübergang. Der rechtselbisch gelegene Brückenkopf Torgau zur Verteidigung der Elbbrücke und damit der Stadt ist die uns geläufige Befestigung, die wir „Brückenkopf“ nennen. Nach der neuen Idee vom „doppelten Brückenkopf“ musste zwangsläufig auch die Kernbefestigung auf der Westseite, also Torgau selbst, als der zweite Brückenkopf definiert werden, d. h. die Hauptfestung Torgau wurde selbst zum Brückenkopf.

Das Konzept des „doppelten Brückenkopfes“ ver-

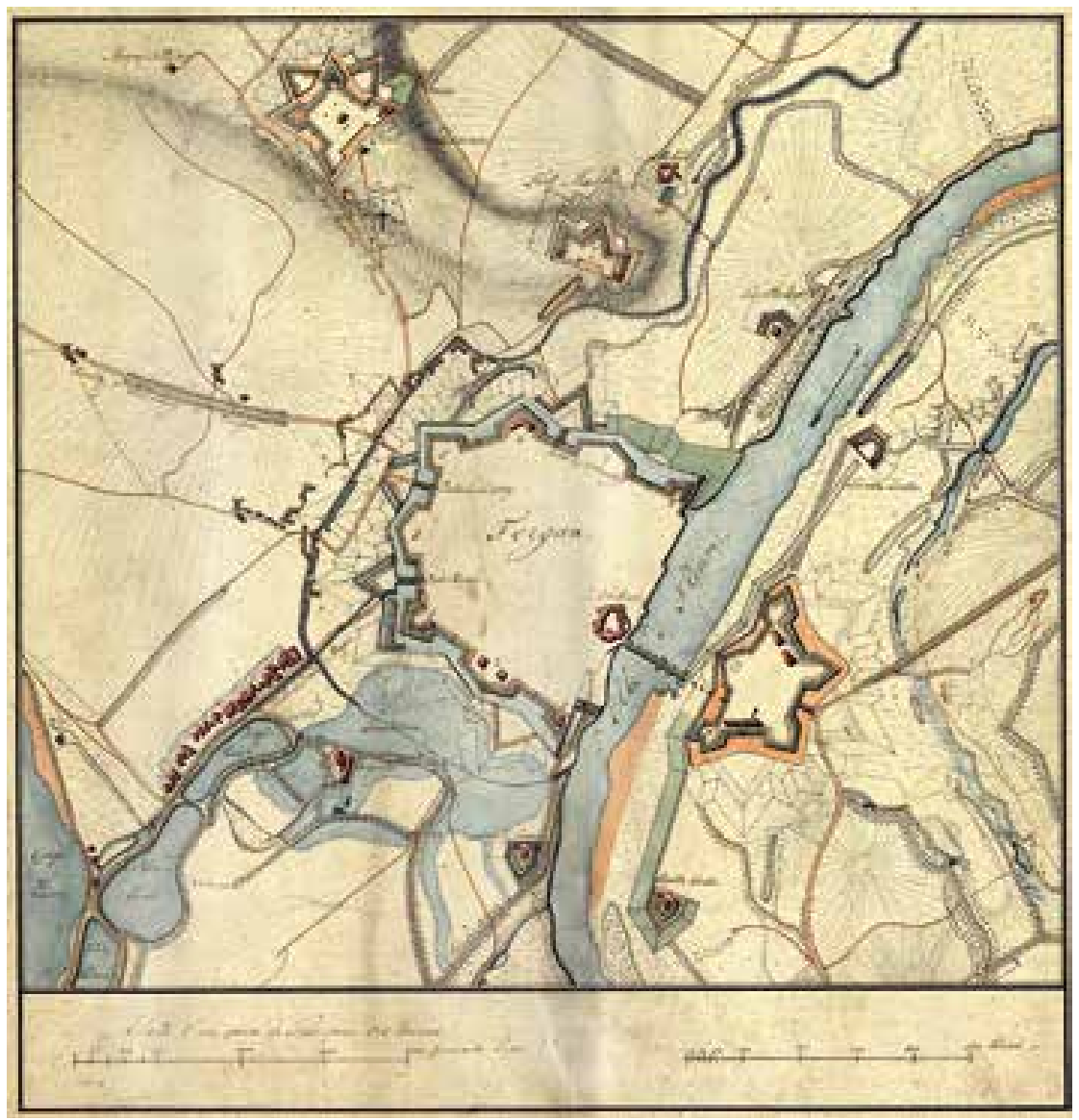

langte bau- und armierungstechnische Voraussetzungen bzw. Veränderungen innerhalb der Festung, war aber vor allem eine militärische Strategie, die auf wechselseitigen Bewegungen der Armeen zu beiden Seiten des Flusses beruhte. Die „KampagneFestung“ war so auszurüsten, dass sowohl die eine wie die andere Seite Brückenkopf sein kann. Zu diesem Konzept gehören mobile Armeeeinheiten, welche auf Abruf wechselseitig auf die jeweils andere Flussseite übergehen konnten. Die Kampagne-Festung gab dieser mobilen Truppe wechselseitig Flankierung und Schutz. Die Truppe war somit in der Lage, einem irgendwo über den Fluss gegangenen Feind unmittelbar nachzusetzen und in dessen Flanke oder deren Rücken zu stoßen.

Um das Vorhaben einer offensiven „Kampagne-Festung" umgehend zu realisieren, strich Napoleon in dem Aster-Memoire alles Mögliche zusammen, aber vor allem wurde aus der von den Sachsen vorgesehenen Festung mit zehn Bastionen eine kleinere mit sechs Bastionen und zwei Halbbastionen. Die Baukosten konnten durch diese Reduzierung verringert und die Bauzeit verkürzt werden. Der Russland-Feldzug sollte ja schon bald beginnen. Allerdings waren dazu die Vorstädte von Torgau einschließlich Kirchen und Hospitäler abzureißen. Selbst die geplanten sächsischen Festungsbauten im Doppelwall wurden ausgedünnt und vereinfacht. Die bombensicheren Schutzräume für die Mannschaft im Festungsinneren, auf die Aster so viel Wert gelegt hatte, entfielen. Und aus einer vorgesehenen Kasernierung der Mannschaft und Truppen wurden vor allem Einquartierungen zu Lasten der Einwohnerschaft. Für eine festungsübliche medizinische Versorgung und für Lazarette hatte man nicht ausreichend gesorgt. Das war eine Auslassung, die sich nach dem Russland-Desaster, 1813, bitter rächte.

In den Gesprächen mit Napoleon war Aster freilich bestrebt, möglichst viele Elemente der von den sächsischen Ingenieuren geplanten Landesfestung Torgau durchzusetzen. Er konnte abwenden, dass die erdgeschüttete bastionäre Umwallung des Hauptwerks direkt auf die mittelalterliche Stadtmauer gelegt wurde. Das hätte bedeutet, dass noch mehr Gebäude in Torgau vor dem Hauptwall abzureißen gewesen wären.

\section{Bau und Nutzung der Festung Torgau 1811 bis 1813}

Napoleons Liste zur Verschlankung der sächsischen Landesfestung zu der genannten Kampagne-Festung wurde von den Sachsen baulich in Angriff genommen und in der geplanten Zeit vollendet. In einem Jahr, 1811, wurde das Hauptwerk mit seinen acht Bastionen erdgeschüttet gebaut. Angelegt waren pfeilförmige Werke (Ravelins) vor den drei Festungstoren sowie eine Kontergarde zur zusätzlichen Verteidigung der Bastion III. Errichtet wurden dann auch zwei Pulvermagazine im Hof der Bastion II. Begonnen wurde mit dem Wallgraben und den Staumauern (Batardeaus) sowie dem Überflutungsbecken (Inundationsbecken), auch wurde die Teichschanze 
vorbereitet. Im Jahr 1812 wurden die Außenwerke errichtet, darunter der östliche Brückenkopf, die vier Elbelünetten, das Fort Zinna und das Fort Mahla.

Die Armierung wurde bedacht und eingeleitet, d.h. die Kanonenrohre und Lafetten bereitgestellt, um nach Ausrufung des Verteidigungszustandes die schon vorbereiteten Artilleriestellungen auf dem Wallgang zu belegen. Anfang Mai 1813 stand die Festung im Wesentlichen. Damals war Johann Adolf Freiherr von Thielmann (1765-1824) Kommandant und Ernst Ludwig Aster Chef des Generalstabes der Festung Torgau, beides sächsische Offiziere. Bald darauf musste die Festung auf Befehl Napoleons und mit Zustimmung des sächsischen Königs unter französisches Kommando gestellt werden.

Als Napoleon am 10. Juli 1813 selbst nach Torgau kam, er die Festungswerke umritt und Revue über die Truppen hielt, „war er sogar freundlich, wie man ihn selten sah. Die Festung hatte ihm gefallen." So jedenfalls schrieb es der Torgauer Ratsherr Niese an seinen Freund Pfarrer Spitzner. Was Napoleon in Torgau gesehen hatte, war das Ergebnis sächsischer Wertarbeit, war eine aufopferungsvolle und fachgerecht ausgeführte Festungsbaukunst des sächsischen Ingenieur-Korps, war die solide Arbeit von sächsischen Soldaten, Handwerkern und vielen Hilfskräften.

Nach der Russland-Niederlage Napoleons kam es $1813 \mathrm{zu}$ einer Konzentration der geschlagenen, zurückströmenden und Zuflucht suchenden Truppen in die Festung Torgau. In der überfüllten Festung brach eine Typhusepidemie aus; insgesamt sollen bis zu 30.000 Soldaten umgekommen sein. Es gab kaum Lazarette in der Stadt. Aufgrund des Mangels an Nahrung war der Hunger stets gegenwärtig. Die Belagerung und das Bombardement durch die Preußen ab dem Herbst 1813, ohne kasemattierte Schutzräume für die Besatzung, ließen katastrophale Verhältnisse in der Stadt entstehen. Jetzt wären die ehemals geplanten Mannschaftsbauten dringend benötigt worden. Die von Napoleon bestimmte Struktur und Funktion der Kampagne-Festung Torgau passte einfach nicht zu der sich plötzlich neu aufgetanen Situation, nämlich Rückzugsraum von Armeeeinheiten einer geschlagenen Armee und Lazarettort für Verwundete zu sein. Der jetzt benötigte, für die Defensive angelegte Festungsumzug war mit Napoleons „offensiver" Auslegung nicht kompatibel. Torgau fiel aufgrund der dramatischen Zuspitzung des Kriegsgeschehens gleich doppelt aus, und zwar einerseits als „offensive“ Fortifikation zur Unterstützung der Kampagne der napoleonischen Feldtruppen und andererseits als ausgedehnte, komplexe und defensiv angelegte Landesfestung mit der Aufgabe, die Truppen, Verwundete und Seuchenkranke aufzunehmen.

\section{Torgau als preußische Festung 1813 bis 1889}

Die Festung Torgau wurde Ende 1813 zur Kapitulation gezwungen. Am 10. Januar 1814 übernahm die preußische Armee das Kommando. König Friedrich Wilhelm III. von Preußen (1770-1840) konnte sein Ziel, das ganze Königreich Sachsen zu übernehmen, durch den Einspruch Österreichs nicht verwirkli- chen. Bei der Teilung Sachsens bestand er aber darauf, die Festungen Torgau und Wittenberg zu erhalten. Somit wurde Torgau gemäß dem Pressburger Vertrag im Mai 1815 auch staatsrechtlich zur preußischen Festung.

Preußens Widerpart im 19. Jahrhundert war unter anderem Österreich, anfangs wiederum auch Sachsen. Die Elbfestung Torgau war 14 Kilometer von der Landesgrenze entfernt. Torgau war, wie auch Wittenberg und Magdeburg, eine preußische Elbfestung, doch aufgrund der Nähe zu Sachsen wurde sogar von der „Preußischen Elb- und Grenzfestung Torgau“ gesprochen.

Die um 1860 verfasste Denkschrift des preußischen Generals Helmuth von Moltke (1800-1891), Chef des Generalstabs, sah vor, den Aufmarschraum der preußischen Armee gegen Österreich und Sachsen durch Festungen, im Besonderen auch die von Torgau, in Verbindung mit natürlichen Hindernissen, hier die Elbe, zu sichern. Und umgekehrt, bei einem etwaigen Angriff der Österreicher auf Berlin, wäre das IV. Preußische Armeekorps innerhalb von 20 Tagen in der Lage, sich bei Torgau mit 20.000 Mann zu versammeln, um eine aktive Flankenverteidigung, die Elbe einbeziehend, auszuführen. Andererseits, bei einem Angriff der Österreicher links der Elbe, könnte die Festung Torgau mit einem den Fluss überquerenden Armeekorps, so Moltkes Denkschrift, als „quer vorgelegte Schranke“ fungieren. Insgesamt kamen die Preußen damals zu dem Schluss, dass die gegen Österreich (und Sachsen) gerichteten Funktionen (Flankenverteidigung; Schranke) zum einen besser durch große Gürtelfestungen wie Torgau, Wittenberg und Magdeburg und zum anderen durch deren komplexes Zusammenwirken, d.h. besonders mit der Möglichkeit wechselseitiger Bewegungen über die Elbe mehre-

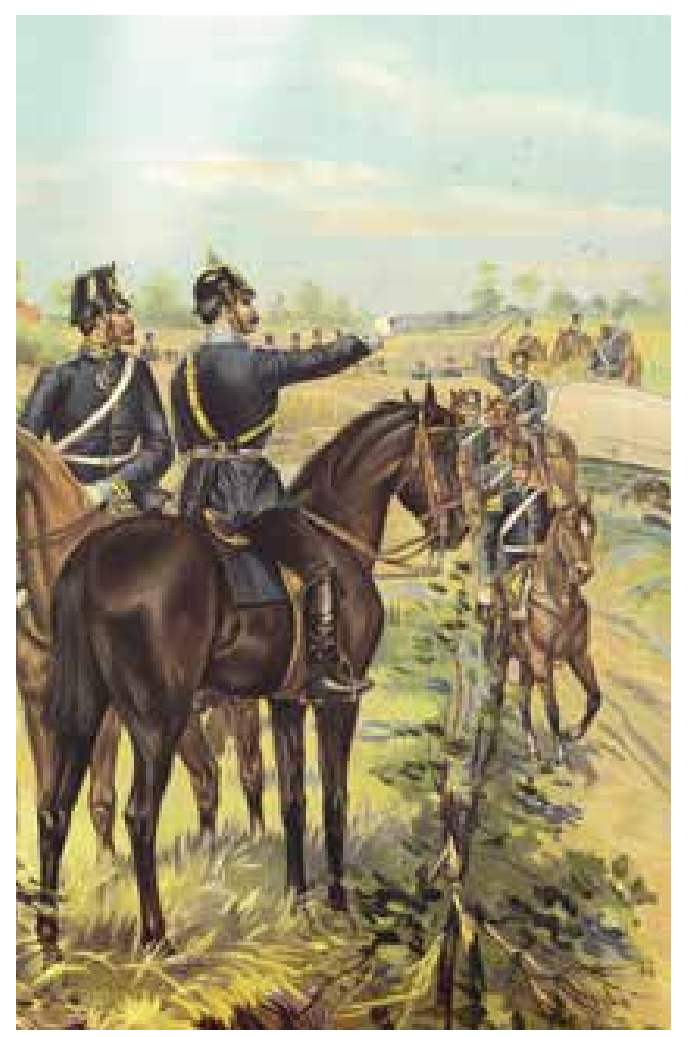

Pontoniere (später wurden sie als Pioniere bezeichnet) bringen ein von einer Transporteinheit des Train herangekarrtes Ponton zu Wasser. Eine Kette von mehreren solchen bootsartigen Schwimmkörpern trug anschließend eine übungsmäßig gebaute Kriegsbrücke. Im Brückenkopf Torgau waren Pioniere stationiert, die den Elbefluss als idealen Wasserübungsplatz zur Ausbildung nutzen konnten. ๑ Förderverein Europa Begegnungen e.V. 
Festung Torgau, Plan von 1888. Auf der ostelbischen Seite, vor dem Brückenkopf-Hauptwall, elbaufwärts, ist ein PionierÜbungsgelände vermerkt, das als Fläche beinahe den gesamten Brückenkopf-Umlauf umgab. ๑ Förderverein Europa Begegnungen e.V.

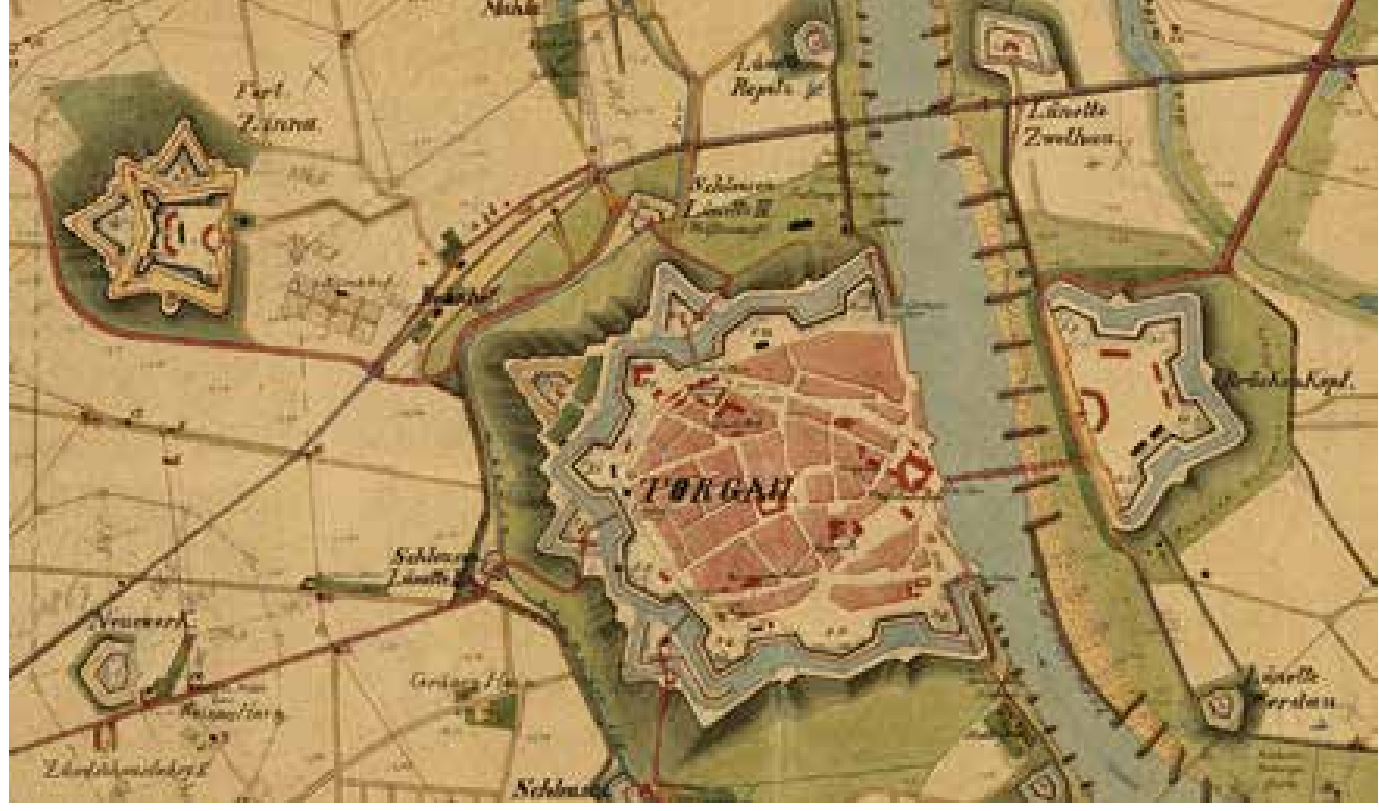

rer preußischer Armeeeinheiten funktionieren sollte. Doch seien für diese Überquerungen zum einen die bestehenden festen Brücken und zum anderen zusätzliche, variabel einsetzbare Pontonübergänge notwendig. Deshalb müsse, so Moltke, die Feldarmee der Preußen mit ausreichenden technischen Truppen und Gerät bestückt und ausgerüstet werden.

Dabei übernahm Moltke von Napoleons Strategie das Konzept der „offensiven“ Festungen, in der In-

terpretation, dass diese ein aktives Element einer vorrangig am Offensivgedanken orientierten Kriegsführung zu sein haben, sowie die Idee eines „doppelten Brückenkopfes“. Darunter verstand man weiterhin befestigte Werke an beiden Flussufern. Doch die Elbübergänge selbst wurden vervielfacht. Pontons bzw. Pontonbrücken, wenn notwendig gleich mehrere Stränge nebeneinander oder in Abständen versetzt über den Fluss geschlagen, hatten die Aufgabe, die Kapazität und Intensi-
In dem Riss des Hauptwerks der Festung Torgau von 1867 sind alle vierzehn Flankenkasematten als kleine dunkle Flächen gut erkennbar. ๑) Förderverein Europa Begegnungen e.V.

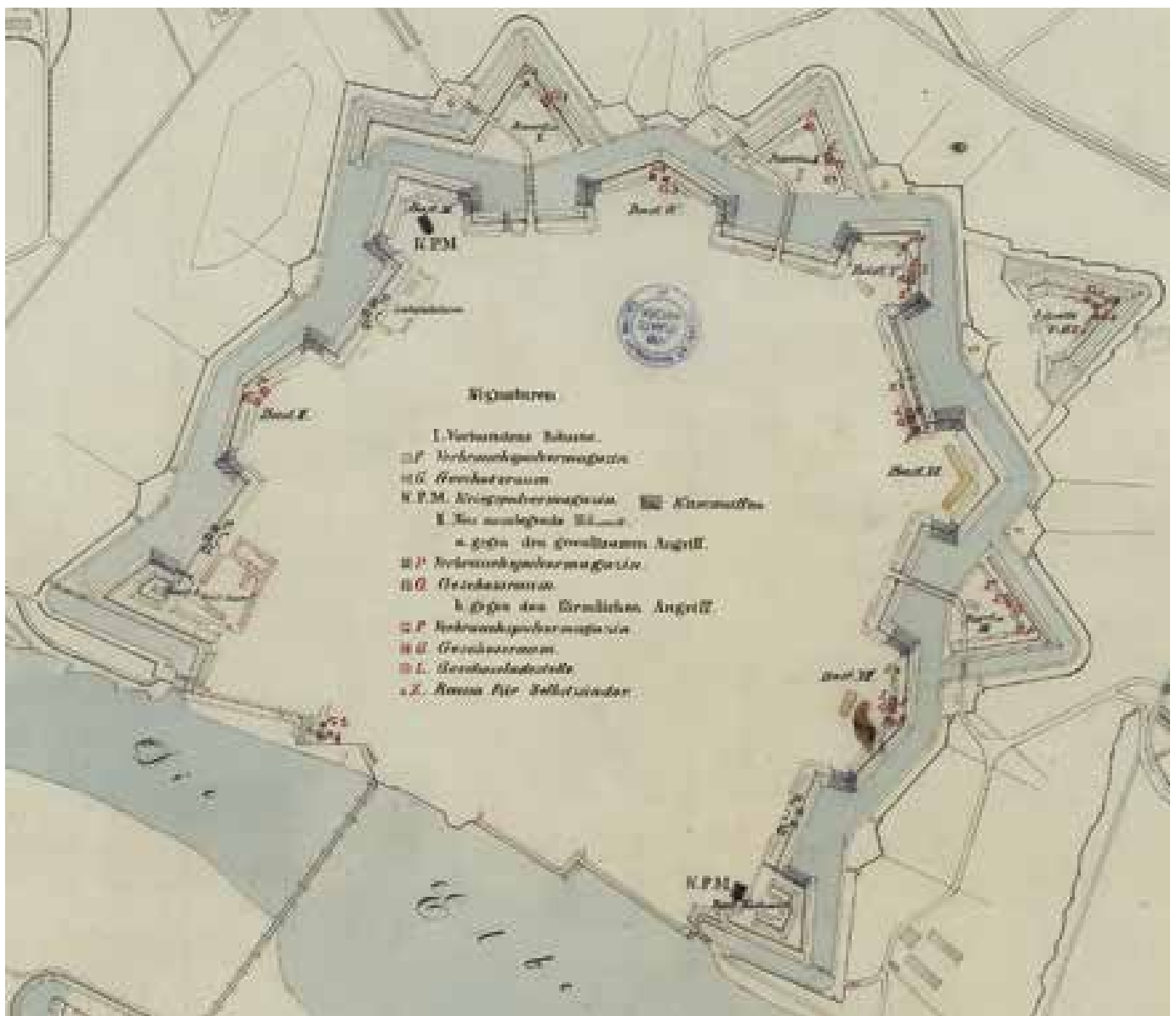


tät beim Übergehen der Truppen zu erhöhen. Einheiten der preußischen Armee trainierten Mitte des 19. Jahrhunderts das Brückenschlagen in einem vorgegebenen Zeitmaß. Die Pontoniere nannten sich dann schon Pioniere. Im Brückenkopf Torgau wurde 1860 das Brandenburgische Pionierbataillon von Rauch Nr. 3 kaserniert. Auf dem südlichen Brückenkopf-Vorfeld, Richtung Kreischau/Werdau, war ein ausgedehnter Pionierübungsplatz errichtet worden.

Moltke und vor allem das preußische Ingenieurkorps bedachten anders als Napoleon, dass eine Festung stets auch Depot, Magazin, Lazarettort, Versammlungsort der Truppe, aber auch Zufluchtsort für geschlagene Armeeeinheiten sein müsse. Als eine der ersten Baumaßnahmen errichteten die Preußen zwischen 1818 und 183014 Flankenkasematten mit einem jeweiligen Kasernenteil, im Hauptwall eingeschlossen, als Schutzraum für die Truppe bei feindlichem Angriff. Eine alte Forderung Asters, mit der er bei Napoleon damals durchgefallen war, wurde jetzt verwirklicht.

Die Festung Torgau war unter den Preußen von Anfang an als Gürtelfestung ausgeführt und aufgrund der sich verbessernden Waffentechnik systematisch erweitert und vervollkommnet worden. Vor allem die Außenwerke wurden in ihrem bisherigen Bestand verbessert oder neu erbaut: drei Festungstore, davor Ravelins am Königstor, Leipziger und Wittenberger Tor, vier Elbelünetten (Repitz, Zwethau, Loßwig und Werdau), Fort Zinna und Fort Mahla; hinzu traten drei Schleusenlünetten; weiter Lünette V/VI; das Neue Werk an der Leipziger Straße; die Teichschanze und die Kuhschanze, um nur die wichtigsten dieser detachierten Werke zu nennen. Sie alle umzogen in angemessenem Abstand das Hauptwerk. Dazwischen ergab sich ein Raum, der als „verschanztes Lager“ für Truppenteile genutzt werden konnte.

Die in anderen Städten fortschreitende industrielle Entwicklung war in Torgau aufgrund der festungsbaulichen Einengung gar nicht möglich. Deshalb ist die historische Altstadt noch heute in beeindruckender Weise erlebbar. Übrigens wurde während der gesamten preußischen Festungszeit von Torgau aus kein einziger abwehrender Kanonenschuss abgefeuert.

\section{Zur Neubebauung Torgaus nach der Schleifung der Festung}

Die Weiterentwicklung der Artillerie machte Befestigungswerke wie in Torgau wirkungslos. Daher wurde 1889 die Festung Torgau aufgehoben und die Entfestigung eingeleitet. Unmittelbar nach der Anordnung der Schleifung setzte die Neubebauung der freigewordenen Festungsabschnitte ein.

Die ersten Überlegungen und Planungen für die Neugestaltung einiger Stadtgebiete hatten in erster Linie den Festungswall-Umzug (Innen- und Außenwall der Bastion I bis VIII) und den zwischen diesen beiden Wällen liegenden Wallgraben zu berücksichtigen. In gleicher Weise waren aber auch der Glacis-Ring mit den feindwärts davor gelege- nen Wasserflächen (Inundationen) wesentlich. Die Inundationen um Torgau herum dehnten sich bis zum Schwarzen Graben aus. Dabei war noch zu beachten, dass drei Ravelins und die Lünette V/VI, die alle in die Glacisfläche hinein ragten, ebenfalls mit niederzulegen waren. Bei den Planungen der Architekten zur Schleifung der Festung wurden in erster Linie der Hauptwall, die Ravelins, die Lünette V/VI und die Glacisfläche der Nord- und der Westseite Torgaus berücksichtigt.

Im Osten war die Neuplanung nicht besonders gravierend, weil hier die Elbe als Wasserhindernis die Stadt begrenzte. Im Süden wurde nach 1894 mit der Errichtung des Elbhafens begonnen. Für den Hafenbau war der gesamte Glacisabschnitt (vor den Bastionen I, II und III) wegzunehmen. An der Elbe und am Hafen gibt es, wie man heute sieht, kein Glacis. Dort ist freilich auch keine Villenbebauung vorhanden. Für den Hafenbetrieb waren die Planung einer Straße und von Gleisen für die Hafenbahn als Transportmöglichkeit wichtig. Eine neue Straße hatte die Elbbrücke Richtung Osten zu erreichen, und sie hatte an die westwärts führenden Ausfallstraßen nach Riesa und Dresden, nach Eilenburg und Leipzig sowie nach Düben bzw. Pretzsch und Wittenberg anzuschließen.

Die Neubebauung Torgaus hatte sich somit auf einen bestimmten Teil des befestigten Umlaufs, nämlich auf die Abschnitte des Festungsringes, auf Wall/Wallgraben, auf Glacis/Inundationen und auf Ravelins/Lünette zu orientieren. Diese lagen im Norden und besonders im Westen der Stadt als Bastionen IV bis VIII. Daraus ist zu folgern, dass wichtige neu zu planende Straßen und die Errichtung der Häuser (Villenbebauung) sich mit dem Nord- und Westring der damaligen Festung Torgau zu befassen hatten. Die Innenstadt Torgaus mit ihren historisch gewachsenen Straßen blieb im Zuge der vorzunehmenden Schleifung weitgehend unberührt.

Bei den Planungen entschied man sich, Ringstraßen auf dem niedergelegten Festungsring mit Ra-

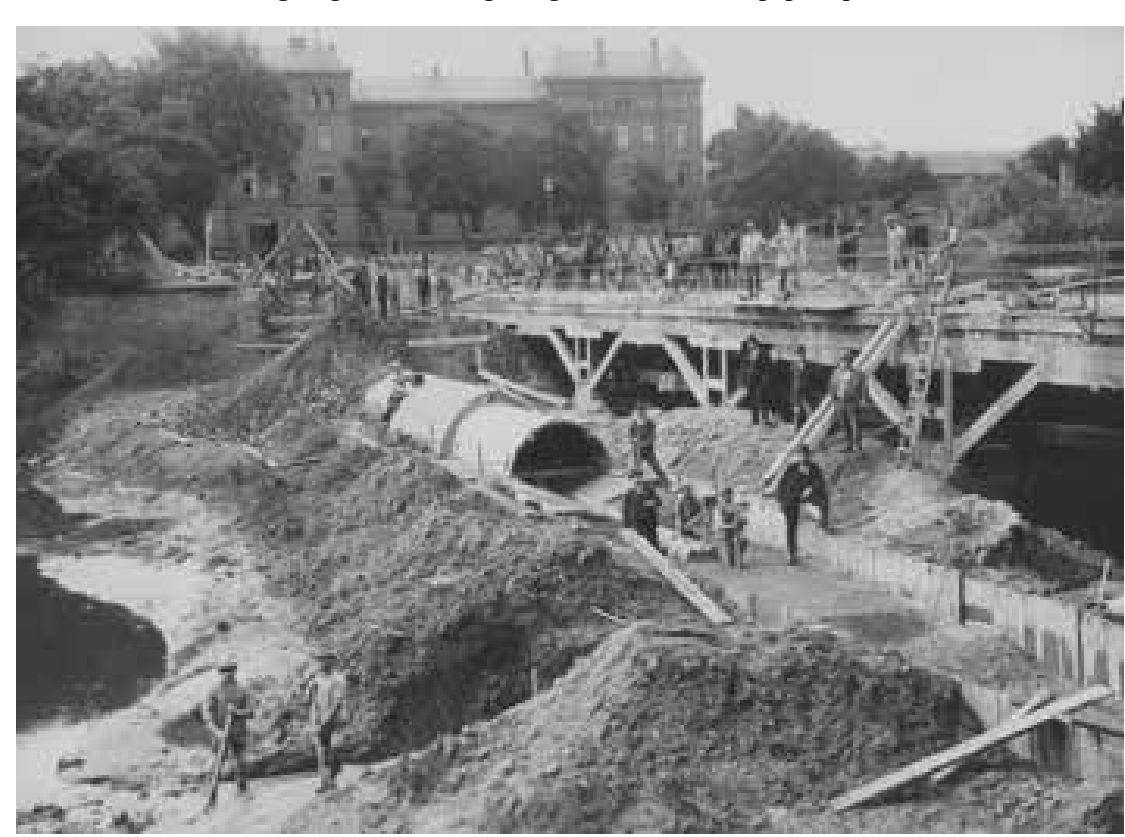

Einbringen der Abwasserkanalisation am ehemaligen Wittenberger Tor um 1900, dahinter die Artilleriekaserne ๑) Förderverein Europa Begegnungen e.V. 


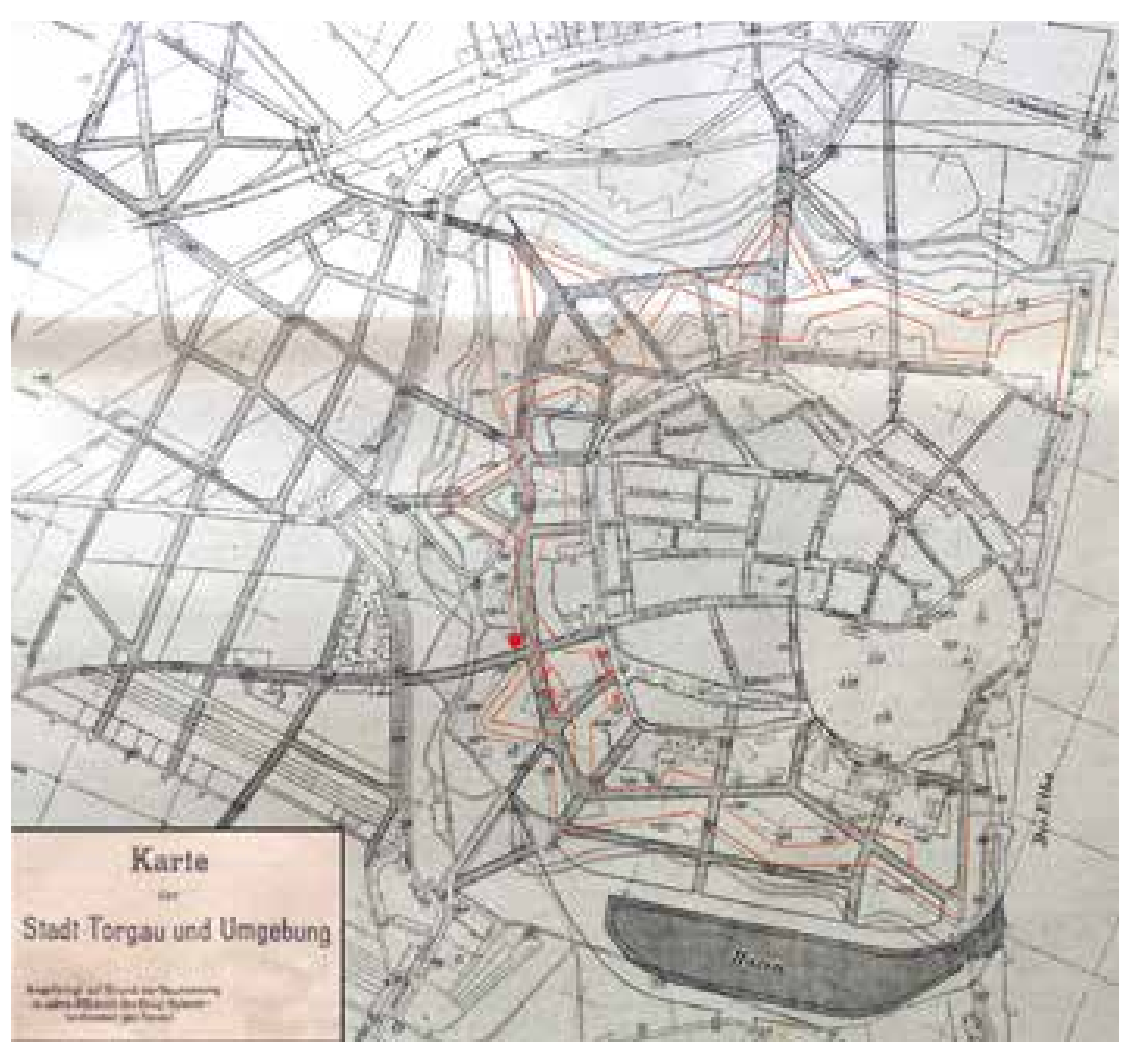

Karte der Stadt Torgau von 1894 mit Eintragung des früheren

Festungsrings und mit den bereits geplanten Straßen, die nach der Schleifung der Wälle realisiert werden sollten.

๑ Förderverein Europa Begegnungen e.V.

Autor

Dr. phil. habil. Uwe Niedersen Förderverein Europa Begegnungen e.V. Schlossstraße 19 04860 Torgau dialstraßen zu verbinden. Eine sehr wichtige Radialstraße war die spätere Bahnhofstraße. Die hatte ihren Ursprung im Wallgraben zwischen den Bastionen $\mathrm{V}$ und VI. Sie teilte dann durch ihre Führung die Lünette V/VI. Die Lünette V/VI war nach 1840 erbaut worden und war ein äußeres Festungswerk mit spitzwinklig zulaufenden Wallschenkeln, die in das Glacis hineinragten. Die neue radiale Straße hatte den Schwarzen Graben und den Gleiskörper der seit 1872 bestehenden Eisenbahn zu überqueren. Sie zog weiter geradlinig in Richtung Fort Zinna und an der um 1900 erbauten Kavallerie-Kaserne vorbei in Richtung Düben und Wittenberg.

Es waren letztlich drei Straßenzüge, die die Neubebauung in Torgau nach 1890 bestimmten: nördlich und westlich waren es Ringstraßen und, wie angemerkt, eine radiale Straße Richtung Bahnhof. Dabei war ein genau zu ermittelndes Höhenniveau bei der Planung des jeweils zu errichtenden Straßendammes wesentlich. Die neuen Straßen hatten eine Höhe zwischen 85 und 86 Meter über N.N. (Normal-Nullpunkt) einzunehmen. Das Auffüllen war aufgrund der Erdmassen, die ja als Festungswälle vorhanden waren, unkompliziert. Die Höhe des Fahrdamms korrespondierte mit der der Glaciskrone, ohne deren Höhe zu erreichen. Die Glaciskrone war die höchste Stelle des Glacis. Von dieser Kante aus dachen sich die Flächen des Glacis bis zu den Inundationen in Richtung Schwarzer Graben allmählich ab. Der Hauptwall wurde mit der Schleifung abgetragen. Mit den Erdmassen verfüllte man den Graben, soweit das notwendig war. Die Glaciskrone um die Stadt war die höchste Linie nach den niedergelegten Wällen. Die Straßendämme dahinter waren stets niedriger. Alle neuen Bebauungen, die sich hinter der Glacis- krone auf der Innenstadtseite befanden, waren hochwassersicher. Bei einem Elbehochwasser mussten Deichbrüche verhindert werden, weshalb man Flutrinnen definierte, die im Stande waren, gefährliche Wassermassen wegzuleiten. Die Flutrinnen waren genau die Glacisflächen, die hinter der Glaciskrone beginnend sich bis zu dem linksseitigen Wallufer des Schwarzen Grabens erstreckten.

Erde zum Auffüllen gab es zur Genüge, und zwar soviel, dass damit auch die Mehrzahl der Wallgräben verfüllt werden konnten. Das Verfüllen des Wallgrabens wurde stets dort vorgenommen, wo die Neubebauung, ob Straßenzug oder Villenbebauung, es verlangte. Natürlich hatte man zuvor dafür zu sorgen, dass die weiten Abwasserrohre und auch die Wasserund Gasleitungen vor dem Verfüllen mitverlegt wurden.

Ein Beispiel für die Berücksichtigung des alten Wallumzugs bei der Stadtplanung bietet der Endabschnitt des Westrings. Der Straßenverlauf stimmte hier abschnittsweise mit dem Wallgrabenumzug vor der Bastion IV überein. Der farbig eingezeichnete Punkt beispielsweise verweist auf den Standort der neuen Villa von G. A. Rabitz jun., Inhaber der neu gegründeten Zündschnurfabrik. Gleich daneben, in Richtung Wasserturmplatz, finden wir hingegen einen wenig verfüllten Wallgrabenabschnitt. Es handelt sich um die mit kleinen roten Kreuzen gekennzeichnete Villenzeile von der verlängerten Leipziger Straße bis zur heutigen Aral-Tankstelle. Die Villen befinden sich alle auf dem niedergelegten inneren Hauptwall zwischen den Bastionen III und IV. Gegenüber liegt die andere Häuserzeile des Südrings. Die Häuser stehen hier auf dem Gegenwall (Kontereskarpe). Dazwischen finden wir, heute noch gut erkennbar, das Profil des Wallgrabens zwischen den Bastionen III und IV, in welchem die Anwohner ihre Gärten angelegt haben.

Alle Straßenabschnitte, vor allem die, welche mit dem verfüllten einstigen Wallgraben-Verlauf übereinstimmten, wurden nicht gleich mit einer Straßenbedeckung (Pflaster) versehen. Über ein Jahr und länger wartete man auf das endgültige Absenken der Verfüllung. Dennoch waren diese Abschnitte zuvor als rohe, unfertige Fahrbahnen für den Verkehr freigegeben.

Alle auf dem Stadtplan von 1894 dunkel schraffierten Straßen waren im Zuge der Schleifung der Festung Torgau geplant worden. Nicht alles Geplante kam zur Ausführung. Das betraf beispielsweise die Straßen, die nördlich der Bastion VII in Richtung Gleiskörper vermerkt sind. Andere auf der Abbildung nicht eingezeichnete Straßen wurden erst später ergänzt, etwa der heutige Martin-Luther-Ring, der direkt auf den abgetragenen Wällen der Bastion VII verläuft.

Über das ganze 19. Jahrhundert hinweg hatten Festungsmauern die Stadt Torgau umklammert. Die Niederlegung der Wälle erbrachte die Möglichkeit, einen begrünten Stadtring mit Villenbebauung anzulegen. Torgau konnte mittels der Neubebauung Versäumtes nachholen sowie mit anderen ähnlichen Städten gleichziehen, teilweise diese sogar noch übertreffen. 